\title{
The 4-loop slope of the Dirac form factor
}

\author{
Stefano Laporta ${ }^{1,2, a, b}$ \\ ${ }^{1}$ Dipartimento di Fisica e Astronomia, Università di Padova, Via Marzolo 8, I-35131 Padova, Italy \\ ${ }^{2}$ Istituto Nazionale Fisica Nucleare, Sezione di Padova, Via Marzolo 8, I-35131 Padova, Italy
}

Abstract. The 4-loop contribution to the slope of the Dirac form factor in QED has been evaluated with 1100 digits of precision. The value is

$$
m^{2} F_{1}^{(4)^{\prime}}(0)=0.886545673946443145836821730610315359390424032660064745 \ldots\left(\frac{\alpha}{\pi}\right)^{4} .
$$

We have also obtained a semi-analytical fit to the numerical value. The expression contains harmonic polylogarithms of argument $e^{\frac{i \pi}{3}}, e^{\frac{2 i \pi}{3}}, e^{\frac{i \pi}{2}}$, one-dimensional integrals of products of complete elliptic integrals and six finite parts of master integrals, evaluated up to 4800 digits. We show the correction to the energy levels of the hydrogen atom due to the slope.

\section{The slope of the Dirac form factor}

In QED the vertex can be written

$$
\bar{u}\left(p_{1}\right)\left(\gamma_{\mu} F_{1}(t)+\frac{\sigma_{\mu v}}{2 m} q_{v} F_{2}(t)\right) u\left(p_{2}\right),
$$

where $m$ is the electron mass, $F_{1}(t)$ and $F_{2}(t)$ are the Dirac and Pauli form factors. At $t=0$, the charge conservation implies that

$$
F_{1}(0)=1,
$$

whereas the value of the Pauli form factor is the $g-2$

$$
F_{2}(0)=\frac{g-2}{2} .
$$

The quantity $\left.\frac{d}{d t} F_{1}(t)\right|_{t=0}=F_{1}^{\prime}(0)$ is the slope of the Dirac form factor.

\subsection{Theoretical expression}

We expand perturbatively the slope in powers of $\left(\frac{\alpha}{\pi}\right)$

$$
m^{2} F_{1}^{\prime}(0)=A_{1}\left(\frac{\alpha}{\pi}\right)+A_{2}\left(\frac{\alpha}{\pi}\right)^{2}+A_{3}\left(\frac{\alpha}{\pi}\right)^{3}+A_{4}\left(\frac{\alpha}{\pi}\right)^{4}+\ldots
$$

The coefficients known in analytical form are [2-4]

$$
\begin{gathered}
A_{1}=-\frac{1}{8}-\frac{1}{6 \epsilon} . \\
A_{2}=-\frac{4819}{5184}-\frac{49}{432} \pi^{2}+\frac{1}{2} \pi^{2} \ln 2-\frac{3}{4} \zeta(3)
\end{gathered}
$$

\footnotetext{
a e-mail: stefano.laporta@ pd.infn.it

${ }^{\mathrm{b}}$ Talk given at "Flavour changing and conserving processes" 2019 (FCCP2019), 29-31 August 2019, Villa Orlandi, Anacapri, Capri, Italy.
}

0. 88654567394644314583682173061031535939042403266006474536805 5909320840316465628927454836486324177336869351275874721830799 6875923974888466826147611753011917584831446774752672980326917 4027192146515393255198447931004950196245313721193729467160800 6342998095842536958494506068383665985141387321894210012394882 7595153823786537220388349644856007568985761687756410271977960 3910290276615122356406105399227905150277608224592369504332757 Q361335093525176476399251682267935964524928545665821844102867 4547644077579921118603788315350119800677785150747802126742479 0405222247330295021831074290199029916276829160228905899116426 4634498789876307270828483643587434780024554153724340089695147 1683115538642559188352093478066512674887503345902599182245563 6131251241198806154155376213371122848462776848674219282896865 6811548030353727600787303621093059264752959892234017835732828 9717496239918335278488413242436969926422136403200684400061242 3529815833966332566753158241741448217616597381276692161976675 0950507406493095613619589880245645116354567571623094417388481 1565020098334847940590188785421700667378220853053541953188378 $610075518116338519220 \ldots$

Table 1. First 1100 digits of $A_{4}$.

$$
=0.469941487459992 \ldots,
$$

$$
\begin{aligned}
A_{3} & =-\frac{17}{24} \pi^{2} \zeta(3)+\frac{25}{8} \zeta(5)-\frac{217}{9}\left(\operatorname{Li}_{4}\left(\frac{1}{2}\right)+\frac{\ln ^{4} 2}{24}\right) \\
& -\frac{103}{1080} \pi^{2} \ln ^{2} 2+\frac{3899}{25920} \pi^{4}-\frac{2929}{288} \zeta(3)+\frac{41671}{2160} \pi^{2} \ln 2 \\
& -\frac{454979}{38880} \pi^{2}-\frac{77513}{186624} \\
& =0.171720018909775 \ldots
\end{aligned}
$$

In this paper we present the result of the calculation of $A_{4}$ with a precision of 1100 digits. The first digits of the result are

$$
A_{4}=0.8865456739464431458368217306103153 \ldots
$$




\begin{tabular}{rll}
\hline loop & $F_{1}^{\prime}(0)$ & \multicolumn{1}{l}{$F_{2}(0)$} \\
\hline 1 & $\infty$ & 0.5 \\
2 & 0.469941487459 & -0.328478965579 \\
3 & 0.171720018909 & 1.181241456587 \\
4 & 0.886545673946 & -1.912245764926 \\
5 & & $6.737(159)$ \\
\hline
\end{tabular}

positive

alternating signs

Table 2. Values of the known contributions to $F_{1}^{\prime}(0)$ and $F_{2}(0)$

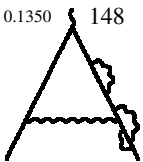

(1)

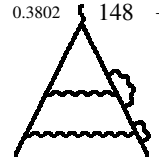

(2)

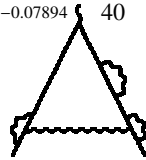

(3)

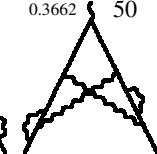

(4)

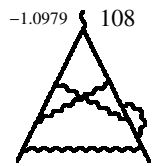

(5)

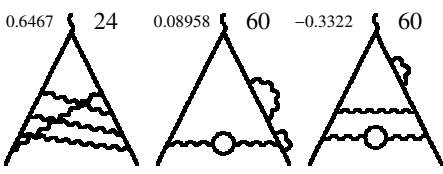

(6)
(7)
(8)

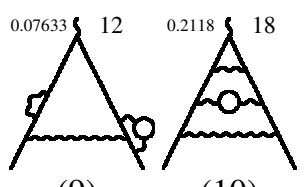

(9)

(10)

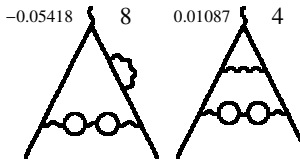

(11)

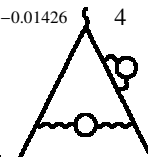

(13)

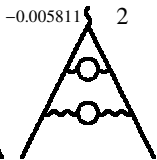

(14)

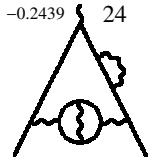

(15)

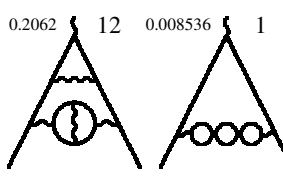

(16)

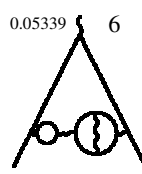

(18)

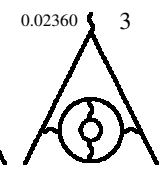

(19)

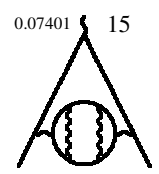

(20)

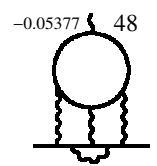

(21)

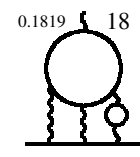

(22)

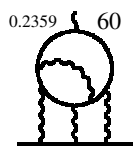

(23)

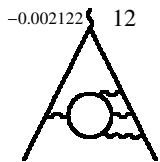

(24)

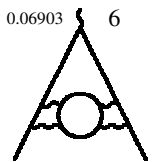

(25)
Figure 1. Typical representative diagrams of gauge-invariant sets. For each set only one diagrams is shown. Top left: contribution of the set to $F_{1}^{\prime}(0)$; top right: number of diagrams of the set

The full-precision result is shown in table 1 . In table 2 we have listed the known values of the slope and $g$-2; we see that $A_{2}, A_{3}$ and $A_{4}$ are all positive, in contrast with the alternating signs observed in the $g$ - 2 .

\subsection{Shift to the hydrogen levels}

Let us now consider the shift to the hydrogen energy levels due to $A_{4}$. We express the energy shift in terms of the frequency shift $\Delta f=\Delta E / h$. For the level $n S$ the frequency shift is $[5,6]$

\begin{tabular}{rr}
\hline 1 & 0.1350531726346435372674724541103838371038 \\
2 & 0.3802929165240844585552528298843579658371 \\
3 & -0.0789488893676831608109628366941799823079 \\
4 & 0.3662786736588470044584250527325325702299 \\
5 & -1.0979832148317652705103820073196531832520 \\
6 & 0.6467871429585372084492391789800382619165 \\
7 & 0.0895891170440342216099366534902414320652 \\
8 & -0.3322086225106643608126657791889571079890 \\
9 & 0.0763376479373933425961220467893817339605 \\
10 & 0.2118669010888818123786340161652003594809 \\
11 & -0.0541837571893361764657206136746826299854 \\
12 & 0.0108761535582321058694530867351119912448 \\
13 & -0.0142646608196830116628021692409901716905 \\
14 & -0.0058117416010420357833143542203438251011 \\
15 & -0.2439068506475319592123409557076293747890 \\
16 & 0.2062012570841125786262218639260170000956 \\
17 & 0.0085366428673036656037790352019835488011 \\
18 & 0.0533927095302949341276880145918233326838 \\
19 & 0.0236058911191014021135877461122766184082 \\
20 & 0.0740163162205724051338179043210727390276 \\
21 & -0.0537711607064956999082765338567906834199 \\
22 & 0.1819474273966664016975772159395176159307 \\
23 & 0.2359289294543601921365690660148707901595 \\
24 & -0.0021225895319909487365222280699442649666 \\
25 & 0.0690362620755704991160330435886767859471 \\
\hline &
\end{tabular}

Table 3. Contribution to $A_{4}$ of the 25 gauge-invariant sets of Fig.1.

$$
\Delta f_{\text {slope }}(n S, 4 \text {-loop })=\frac{4(Z \alpha)^{4} m c^{2}}{h n^{3}}\left(\frac{m_{r}}{m}\right)^{3}\left[\left(\frac{\alpha}{\pi}\right)^{4} A_{4}\right],
$$

where $m_{r}$ is the reduced mass $m_{r}=m M /(m+M)$ and $M$ is the proton mass. Inserting the values of $m, M, c, h$ and $Z=1$, the correction due to $A_{4}$ is

$$
\Delta f_{\text {slope }}(n S, 4-\text { loop })=\frac{36.11}{n^{3}} \mathrm{~Hz},
$$

and is comparable with the experimental error of the extremely precise measurement of $1 \mathrm{~S}-2 \mathrm{~S}$ transition[7]

$$
f(1 \mathrm{~S}-2 \mathrm{~S})=2466061413187018 \pm 11 \mathrm{~Hz} .
$$

Eq.(10) is the first calculated four-loop correction to the energy levels, of the kind $\left(\frac{\alpha}{\pi}\right)^{4}(Z \alpha)^{4}$.

\section{Gauge-invariant sets}

There are 891 vertex diagrams contributing to $A_{4}$. These vertex diagrams can be arranged in 25 gauge-invariant sets (Fig.1). The sets are classified according to the number of photon corrections on the same side of the main electron line and the insertions of electron loops (see Ref.[8]). The numerical contributions of each set, truncated to 40 digits, are listed in the table 3. The separate contributions to the slope from diagrams without or with internal loops are listed in table 4. 


\begin{tabular}{rrl}
\hline loop & $F_{1}^{\prime}(0)$ no elec. loop & $F_{1}^{\prime}(0)$ only elec. loop \\
\hline 2 & 0.438352514986 & 0.031588972473 \\
3 & -0.002437444568 & 0.174157463478 \\
4 & 0.351479801576 & 0.535065872369 \\
\hline
\end{tabular}

Table 4. Separate contributions to the slope from diagrams without and with electron loops.

\section{The analytical fit}

By building systems of integration-by-parts identities[9, $10]$ and solving them[11], the contributions to $A_{4}$ of all the diagrams are expressed as linear combinations of 334 master integrals, the same ones as appeared in the calculation of 4-loop $g$-2 [1]. In Ref.[1] these master integrals were calculated numerically with precision ranging from 1100 to 9600 digits; analytical expressions were fit to all these master integrals (single or in particular combinations) by using the PSLQ algorithm[12, 13]. We use those results here.

Therefore, the analytical expression of $A_{4}$ contains the same transcendentals appeared in the $g$ - 2 result: values of harmonic polylogarithms[14] with argument $1, \frac{1}{2}, e^{\frac{i \pi}{3}}$, $e^{\frac{2 i \pi}{3}}, e^{\frac{i \pi}{2}}[15,16]$, a family of one-dimensional integrals of products of elliptic integrals, and the finite terms of the $\epsilon$-expansions of six master integrals belonging to topologies 24 and 25 of Fig.1. The expression of the analytical fit is written as follows:

$$
A_{4}=T+\sqrt{3} V_{a}+V_{b}+W+\sqrt{3} E_{a}+E_{b}+U,
$$

$$
\begin{aligned}
T= & -\frac{92473962293}{19752284160}-\frac{6619898477}{21772800} \zeta(2)-\frac{12334741}{132300} \zeta(3) \\
& +\frac{97832509}{90720} \zeta(2) \ln 2-\frac{241619904061}{391910400} \zeta(4) \\
& +\frac{4572662443}{12247200} \ln ^{2} 2 \zeta(2)-\frac{1449791143}{3061800}\left(a_{4}+\frac{1}{24} \ln ^{4} 2\right) \\
& +\frac{90355973}{134400} \zeta(5)+\frac{1173056009}{9072000} \zeta(3) \zeta(2)-\frac{8548241}{30240} \zeta(4) \ln 2 \\
& -\frac{68168}{135}\left(a_{5}+\frac{1}{12} \zeta(2) \ln ^{3} 2-\frac{1}{120} \ln ^{5} 2\right)-\frac{244603373713}{52254720} \zeta(6) \\
& -\frac{8082848863}{24192000} \zeta^{2}(3)+\frac{26062}{27} a_{6}-\frac{18215}{27} b_{6}+\frac{18215}{27} a_{5} \ln 2 \\
& -\frac{18215}{27} \zeta(5) \ln 2+\frac{402152509}{189000} a_{4} \zeta(2)+\frac{159693503}{72000} \zeta(3) \zeta(2) \ln 2 \\
& -\frac{328317209}{302400} \zeta(4) \ln ^{2} 2-\frac{18215}{162} \zeta(3) \ln ^{3} 2+\frac{188648503}{1512000} \zeta(2) \ln ^{4} 2 \\
& -\frac{21671}{6480} \ln ^{6} 2-\frac{7224951103}{1741824} \zeta(7)-\frac{1267114025}{387072} \zeta(4) \zeta(3) \\
& -\frac{427145}{504} a_{4} \zeta(3)-\frac{2749470791}{387072} \zeta(5) \zeta(2)+\frac{1420289}{180} a_{5} \zeta(2) \\
& +\frac{116987}{21} a_{7}-\frac{116987}{63} b_{7}+\frac{256321}{756} d_{7}+\frac{971827}{128} \zeta(6) \ln 2 \\
& +\frac{607282}{189} a_{6} \ln 2-\frac{256321}{378} b_{6} \ln _{2} 2-\frac{1794247}{3456} \zeta^{2}(3) \ln 2 \\
& +\frac{104041}{20} a_{4} \zeta(2) \ln 2-\frac{1888991}{24192} \zeta(5) \ln ^{2} 2 \\
& +\frac{256321}{378} a_{5} \ln 22-\frac{9699379}{6048} \zeta(4) \ln ^{3} 2-\frac{2574883}{36288} \zeta(3) \ln ^{4} 2 \\
& +\frac{37144753}{226800} \zeta(2) \ln ^{5} 2-\frac{218465}{127008} \ln ^{7} 2+\frac{75222353}{60480} \zeta(3) \zeta(2) \ln ^{2} 2, \\
&
\end{aligned}
$$

$$
\begin{aligned}
W= & -\frac{1117}{36} \zeta(2) \mathrm{Cl}_{2}\left(\frac{\pi}{2}\right)+\frac{38424}{125} \zeta(2) \mathrm{Cl}_{2}^{2}\left(\frac{\pi}{2}\right) \\
& -118\left(4 \operatorname{Re} H_{0,1,0,1,1}(i) \zeta(2)+4 \operatorname{Im} H_{0,1,1}(i) \mathrm{Cl}_{2}\left(\frac{\pi}{2}\right) \zeta(2)\right. \\
& \left.-2 \mathrm{Cl}_{4}\left(\frac{\pi}{2}\right) \zeta(2) \pi+\mathrm{Cl}_{2}^{2}\left(\frac{\pi}{2}\right) \zeta(2) \ln 2\right),
\end{aligned}
$$

$$
\begin{aligned}
V_{a}= & -\frac{14186171}{194400} \mathrm{Cl}_{4}\left(\frac{\pi}{3}\right)-\frac{103023803}{583200} \zeta(2) \mathrm{Cl}_{2}\left(\frac{\pi}{3}\right) \\
& +\frac{916598}{76545} \operatorname{Im} H_{0,0,0,1,-1,-1}\left(e^{i \frac{\pi}{3}}\right)+\frac{916598}{76545} \operatorname{Im} H_{0,0,0,1,-1,1}\left(e^{i \frac{2 \pi}{3}}\right) \\
& +\frac{916598}{76545} \operatorname{Im} H_{0,0,0,1,1,-1}\left(e^{i \frac{2 \pi}{3}}\right)+\frac{458299}{36855} \operatorname{Im} H_{0,0,1,0,1,1}\left(e^{i \frac{2 \pi}{3}}\right) \\
& +\frac{10540877}{442260} \operatorname{Im} H_{0,0,0,1,1,1}\left(e^{i \frac{2 \pi}{3}}\right)+\frac{178619489}{3980340} \mathrm{Cl}_{6}\left(\frac{\pi}{3}\right) \\
& +\frac{1833196}{45927} a_{4} \mathrm{Cl}_{2}\left(\frac{\pi}{3}\right)-\frac{12563350487}{2579260320} \zeta(5) \pi \\
& +\frac{533401067}{459270} \zeta(4) \mathrm{Cl}_{2}\left(\frac{\pi}{3}\right)+\frac{844343}{18900} \operatorname{Im} H_{0,1,1,-1}\left(e^{i \frac{2 \pi}{3}}\right) \zeta(2) \\
& +\frac{844343}{28350} \operatorname{Im} H_{0,1,1,-1}\left(e^{i \frac{\pi}{3}}\right) \zeta(2)+\frac{458299}{21870} \zeta(3) \operatorname{Im} H_{0,1,-1}\left(e^{i \frac{\pi}{3}}\right) \\
& +\frac{458299}{14580} \zeta(3) \operatorname{Im} H_{0,1,1}\left(e^{i \frac{2 \pi}{3}}\right)-\frac{263673944}{295245} \mathrm{Cl}_{4}\left(\frac{\pi}{3}\right) \zeta(2) \\
& +\frac{39924629}{6889050} \zeta(3) \zeta(2) \pi+\frac{844343}{1224720} \zeta(4) \pi \ln 2 \\
& -\frac{844343}{11340} \operatorname{Im} H_{0,1,-1}\left(e^{i \frac{\pi}{3}}\right) \zeta(2) \ln 2+\frac{19130869}{367416} \zeta(2) \mathrm{Cl}_{2}\left(\frac{\pi}{3}\right) \ln ^{2} 2 \\
& -\frac{844343}{7560} \operatorname{Im} H_{0,1,1}\left(e^{i \frac{2 \pi}{3}}\right) \zeta(2) \ln 2+\frac{458299}{275562} \mathrm{Cl}_{2}\left(\frac{\pi}{3}\right) \ln ^{4} 2,(15)
\end{aligned}
$$

$$
\begin{aligned}
V_{b}= & \frac{212671}{2400}\left(\operatorname{Re}_{0,0,0,1,0,1}\left(e^{i \frac{\pi}{3}}\right)+\mathrm{Cl}_{4}\left(\frac{\pi}{3}\right) \mathrm{Cl}_{2}\left(\frac{\pi}{3}\right)\right) \\
& -\frac{1031987}{14400} \mathrm{Cl}_{2}^{2}\left(\frac{\pi}{3}\right) \zeta(2)-\frac{507}{4} \operatorname{Re} H_{0,0,0,1,0,1,-1}\left(e^{i \frac{\pi}{3}}\right) \\
& -507 \operatorname{Re}_{0,0,0,0,1,1,-1}\left(e^{i \frac{\pi}{3}}\right)+\frac{13689}{32} \operatorname{Re} H_{0,0,1,0,0,1,1}\left(e^{i \frac{2 \pi}{3}}\right) \\
& +\frac{68445}{64} \operatorname{Re} H_{0,0,0,1,0,1,1}\left(e^{i \frac{2 \pi}{3}}\right)+\frac{13689}{8} \operatorname{Re} H_{0,0,0,0,1,1,1}\left(e^{i \frac{2 \pi}{3}}\right) \\
& -\frac{507}{4} \mathrm{Cl}_{4}\left(\frac{\pi}{3}\right) \operatorname{Im} H_{0,1,-1}\left(e^{i \frac{\pi}{3}}\right)-\frac{1521}{8} \mathrm{Cl}_{4}\left(\frac{\pi}{3}\right) \operatorname{Im} H_{0,1,1}\left(e^{i \frac{2 \pi}{3}}\right) \\
& -\frac{24505}{176} \mathrm{Cl}_{6}\left(\frac{\pi}{3}\right) \pi-\frac{295}{4} \operatorname{Re}_{0,1,0,1,-1}\left(e^{i \frac{\pi}{3}}\right) \zeta(2) \\
& -\frac{295}{2} \operatorname{Re}_{0,0,1,1,-1}\left(e^{i \frac{\pi}{3}}\right) \zeta(2)-\frac{2655}{16} \operatorname{Re}_{0,1,0,1,1}\left(e^{i \frac{2 \pi}{3}}\right) \zeta(2) \\
& -\frac{2655}{8} \operatorname{Re}_{0,0,1,1,1}\left(e^{i \frac{2 \pi}{3}}\right) \zeta(2)-\frac{295}{4} \operatorname{Im}_{0,1,-1}\left(e^{i \frac{\pi}{3}}\right) \mathrm{Cl}_{2}\left(\frac{\pi}{3}\right) \zeta(2) \\
& -\frac{885}{8} \operatorname{Im} H_{0,1,1}\left(e^{i \frac{2 \pi}{3}}\right) \mathrm{Cl}_{2}\left(\frac{\pi}{3}\right) \zeta(2),
\end{aligned}
$$

$$
\begin{aligned}
E_{a}= & \pi\left(\frac{5581729229}{362880000} B_{3}+\frac{1233637481}{1399680000} C_{3}\right) \\
& -\frac{11495611}{3265920} \pi f_{2}(0,0,1)+\pi\left(\frac{751}{972} \ln 2 f_{2}(0,0,1)\right. \\
& -\frac{365478661}{24494400} f_{2}(0,2,0)+\frac{119022487}{5443200} f_{2}(0,1,1) \\
& \left.-\frac{119022487}{14515200} f_{2}(0,0,2)\right)-\frac{751}{729} \zeta(2) f_{1}(0,0,1) \\
& +\pi\left(-\frac{1735283}{497664} \zeta(2) f_{2}(0,0,1)+\frac{1105}{108} \ln 2 f_{2}(0,0,2)\right. \\
& -\frac{2210}{81} \ln 2 f_{2}(0,1,1)+\frac{4420}{243} \ln 2 f_{2}(0,2,0)-\frac{1104271}{497664} f_{2}(0,0,3) \\
& +\frac{272833}{41472} f_{2}(0,1,2)-\frac{4011005}{497664} f_{2}(0,2,1)+\frac{8417635}{2239488} f_{2}(0,3,0) \\
& +\frac{157753}{248832} f_{2}(1,0,2)+\frac{354323}{248832} f_{2}(1,1,1)-\frac{298711}{124416} f_{2}(1,2,0) \\
& \left.-\frac{157753}{497664} f_{2}(2,0,1)-\frac{98285}{248832} f_{2}(2,1,0)\right),
\end{aligned}
$$




\begin{tabular}{rr}
\hline$T$ & -2191.23546965751178841316292285882885509 \\
$\sqrt{3} V_{a}$ & -648.74441479274053140037234999290048941 \\
$V_{b}$ & -400.66449515766079257160481868291283752 \\
$W$ & 1539.32919916681645350981276108756905937 \\
$\sqrt{3} E_{a}$ & -266.54091710106238111286732183079933994 \\
$E_{b}$ & 1928.22253648844241548541655379066123429 \\
$U$ & 40.52010672766306764861492021782154366 \\
\hline
\end{tabular}

Table 5. Numerical values of the addends appearing in Eq.12.

$$
\begin{aligned}
E_{b}= & \zeta(2)\left(-\frac{4629335}{165888} f_{1}(0,0,2)+\frac{112357}{1536} f_{1}(0,1,1)-\frac{99731}{1944} f_{1}(0,2,0)\right. \\
& \left.+\frac{157753}{41472} f_{1}(1,0,1)\right)
\end{aligned}
$$

$$
\begin{aligned}
U= & \frac{174623}{288000} C_{81 a}+\frac{29479}{7200} C_{81 b}-\frac{43}{6} C_{81 c}+\frac{10871}{14400} C_{83 a}-\frac{157}{1620} C_{83 b} \\
& -\frac{95}{24} C_{83 c} .
\end{aligned}
$$

In the above expressions $\zeta(n)=\sum_{i=1}^{\infty} i^{-n}, a_{n}=$ $\sum_{i=1}^{\infty} 2^{-i} i^{-n}, b_{6}=H_{0,0,0,0,1,1}\left(\frac{1}{2}\right), b_{7}=H_{0,0,0,0,0,1,1}\left(\frac{1}{2}\right)$, $d_{7}=H_{0,0,0,0,1,-1,-1}(1), \mathrm{Cl}_{n}(\theta)=\operatorname{ImLi}_{n}\left(e^{i \theta}\right) . H_{i_{1}, i_{2}, \ldots}(x)$ are the harmonic polylogarithms. The integrals $f_{j}$ are defined as follows:

$$
\begin{aligned}
f_{m}(i, j, k)= & \int_{1}^{9} d s D_{1}(s) \operatorname{Re}\left(\sqrt{3^{m-1}} D_{m}(s)\right) \times \\
D_{m}(s)= & \frac{2}{\sqrt{(\sqrt{s}+3)(\sqrt{s}-1)^{3}}} \times \ln ^{i}(9-s) \ln ^{j}(s-1) \ln ^{k}(s), \\
& K\left(m-1-(2 m-3) \frac{(\sqrt{s}-3)(\sqrt{s}+1)^{3}}{(\sqrt{s}+3)(\sqrt{s}-1)^{3}}\right)
\end{aligned}
$$

$K(x)$ is the complete elliptic integral of the first kind. The constants $B_{3}$ and $C_{3}$ have the following hypergeometric representations $[1,17]$ :

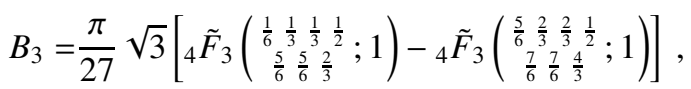

$$
\begin{aligned}
& C_{3}=\frac{\pi}{27} \sqrt{3}\left[{ }_{4} \tilde{F}_{3}\left(\begin{array}{ccc}
\frac{1}{6} \frac{1}{3} \frac{4}{3}-\frac{1}{2} \\
-\frac{1}{6} \frac{5}{6} \frac{5}{3}
\end{array} 1\right)-{ }_{4} \tilde{F}_{3}\left(\begin{array}{c}
-\frac{7}{6}-\frac{1}{3} \frac{2}{3}-\frac{1}{2} \\
-\frac{5}{6} \frac{1}{6} \frac{1}{3}
\end{array} ; 1\right)\right] \text {, }
\end{aligned}
$$

${ }_{4} \tilde{F}_{3}\left(\begin{array}{c}a_{1} a_{2} a_{3} a_{4} \\ b_{1} b_{2} b_{3}\end{array} ; x\right)=\frac{\Gamma\left(a_{1}\right) \Gamma\left(a_{2}\right) \Gamma\left(a_{3}\right) \Gamma\left(a_{4}\right)}{\Gamma\left(b_{1}\right) \Gamma\left(b_{2}\right) \Gamma\left(b_{3}\right)}{ }_{4} F_{3}\left(\begin{array}{c}a_{1} a_{2} a_{3} a_{4} \\ b_{1} b_{2} b_{3}\end{array} ; x\right)$.

The numerical values of the constants appearing in Eq.(12) are listed in Table 5. Note the strong numerical cancellations in Eq.(12): the largest term is $-\frac{2749470791}{387072} \zeta(2) \zeta(5)=$ -12115.862 .

\section{Method of calculation}

We sketch the method used to obtain $A_{4}$. It is the same used in Ref.[1].

1. Generation of 891 vertex diagrams ( $C$ program) from 104 self-mass diagrams. These are the same of the 4-loop $g$-2 calculation.

2. Extraction of the contribution to $A_{4}$ from the amplitude of each diagram by using projectors $[18,19]$ with a FORM program[20, 21].

3. Algebraic reduction to master integrals, obtained by building and solving large systems of integrationby-parts identities[9, 10] by using the program SYS[11].

4. For the sake of checks we generate a different system for each group of vertex diagrams obtained from the same self-mass diagram.

5. The smallest system contains $10^{8}$ identities, with size of $90 \mathrm{~GB}$. The system with the largest number of identities contains $5 \times 10^{8}$, with a size of $170 \mathrm{~GB}$. The largest system has $3 \times 10^{8}$ identities with a size of $1.2 \mathrm{~TB}$.

6. The ratio between number of independent identities and total number of generated identities is in the range $0.2-0.3$. The dependent identities become trivial zeroes when substituted into the system, and have been used to check the reliability of hardware and software. No hardware errors were detected. Instead, software errors have been detected in this way (frequency: one every 2-3 weeks), caused by a bug in the OpenMPI message passing library used with the highest level of threads support.

7. We algebraically check that the contribution from a diagram is invariant to the changes in the particular internal routing of the momentum of the external photon.

8. The renormalization is carried out by subtracting suitable counterterms, which are generated with $\mathrm{C}$ and FORM programs and calculated numerically with SYS.

\section{Acknowledgments}

This work has been supported by the Supporting TAlent in ReSearch at Padova University (UniPD STARS Grant 2017 "Diagrammalgebra”).

I wish to thank Pierpaolo Mastrolia for the encouragement and the support. I wish to thank Thomas Gehrmann for providing me the access to the computing facilities of the Institute for Theoretical Physics of Zurich. 


\section{References}

[1] S. Laporta, Phys. Lett. B 772 (2017) 232

[2] R. Barbieri, J. A. Mignaco and E. Remiddi, Nuovo Cim. A 11 (1972) 824.

[3] R. Barbieri, J. A. Mignaco and E. Remiddi, Nuovo Cim. A 11 (1972) 865.

[4] K. Melnikov and T. van Ritbergen, Phys. Rev. Lett. 84 (2000) 1673.

[5] G. W. Erickson and D. R. Yennie, Annals Phys. 35, (1965) 271.

[6] G. W. Erickson and D. R. Yennie, Annals Phys. 35, (1965) 447.

[7] A. Matveev et al., Phys. Rev. Lett. 110 (2013) no.23, 230801.

[8] P. Cvitanovic, Nucl. Phys. B 127 (1977) 176.

[9] K. G. Chetyrkin and F. V. Tkachov, Nucl. Phys. B 192 (1981) 159.

[10] F. V. Tkachov, Phys. Lett. 100B (1981) 65.

[11] S. Laporta, Int. J. Mod. Phys. A 15 (2000) 5087
[12] H. R. P. Ferguson and D. H. Bailey, RNR Technical Report RNR-91-032.

[13] D. H. Bailey and D. J. Broadhurst, Math. Comput. 70 (2001) 1719

[14] E. Remiddi and J. A. M. Vermaseren, Int. J. Mod. Phys. A15 (2000) 725.

[15] S. Laporta, PoS LL $2018 \quad$ (2018) 073. doi:10.22323/1.303.0073

[16] J. Ablinger, J. Blumlein and C. Schneider, J. Math. Phys. 52 (2011) 102301

[17] Y. Zhou, Ramanujan J. (2019). https://doi.org /10.1007/s11139-018-0090-z

[18] R. Barbieri, M. Caffo, E. Remiddi, S. Turrini and D. Oury, Nucl. Phys. B 144 (1978) 329.

[19] S. Laporta and E. Remiddi, Adv. Ser. Direct. High Energy Phys. 20 (2009) 119.

[20] J. A. M. Vermaseren, arXiv:math-ph/0010025

[21] J. Kuipers, T. Ueda, J. A. M. Vermaseren and J. Vollinga, Comput. Phys. Commun. 184 (2013) 1453 